INPLASY

PROTOCOL

To cite: Li et al. Prognostic and Clinicopathologic Significance of Neutrophil-to-lymphocyte

Ratio in Esophageal Cancer:

An Update Meta-analysis.

Inplasy protocol 2021100111.

doi:

10.37766/inplasy2021.10.0111

Received: 28 October 2021

Published: 28 October 2021

Corresponding author:

Sheng Wang

swangcancr@163.com

Support: Private ownership.

Review Stage at time of this submission: Data analysis Completed but not published.

Conflicts of interest:

None declared.

\section{Prognostic and Clinicopathologic Significance of Neutrophil-to- lymphocyte Ratio in Esophageal Cancer: An Update Meta-analysis}

\author{
Li, B1; Xiong, F2; Yi, S3; Wang, S4.
}

Review question / Objective: Assess the prognostic and clinicopathologic significance of Neutrophil-to-lymphocyte Ratio (NLR) in patients with esophageal cancer.

Condition being studied: Prognosis and clinicopathologic characteristics of esophageal cancer.

Eligibility criteria: 1) esophageal cancer was diagnosed with pathology; 2) NLR was measured before treatment and from serum and with a clear cutoff value; 3 ) the association between NLR and prognosis (OS, CSS, PFS, and DFS) or clinicopathologic parameters were evaluated.

INPLASY registration number: This protocol was registered with the International Platform of Registered Systematic Review and Meta-Analysis Protocols (INPLASY) on 28 October 2021 and was last updated on 28 October 2021 (registration number INPLASY2021100111).

\section{INTRODUCTION}

Review question / Objective: Assess the prognostic and clinicopathologic significance of Neutrophil-to-lymphocyte Ratio (NLR) in patients with esophageal cancer.

Condition being studied: Prognosis and clinicopathologic characteristics of esophageal cancer.

\section{METHODS}

Participant or population: Patients who were diagnosed with esophageal cancer.

Intervention: None.

Comparator: None.

Study designs to be included: a MetaAnalysis. 
Eligibility criteria: 1) esophageal cancer was diagnosed with pathology; 2) NLR was measured before treatment and from serum and with a clear cutoff value; 3 ) the association between NLR and prognosis ( OS, CSS, PFS, and DFS) or clinicopathologic parameters were evaluated.

Information sources: A systematic literature search was performed using PubMed, Embase, and Web of Science up to January 12, 2020.

Main outcome(s): Overall survival of esophageal cancer.

Quality assessment / Risk of bias analysis: Publication bias was assessed using Begg's funnel plot and Egger's linear regression test.

Strategy of data synthesis: HRs and 95\% Cls were used to analyze the relationship between NLR and prognosis (OS, CSS, PFS, and DFS); if univariate and multivariate analyses were both reported in the same study, multivariate-adjusted HRs and $95 \%$ confidence intervals were used in preference. ORs and $95 \% \mathrm{Cls}$ were used to evaluate the relationship between NLR and clinicopathologic factors.

Subgroup analysis: Subgroup analysis was used to explore possible heterogeneity.

Sensitivity analysis: Robustness of the study was tested by sensitivity analysis

Country(ies) involved: China.

Keywords: esophageal cancer; inflammation; meta-analysis; neutrophilto-lymphocyteratio.

Contributions of each author:

Author 1 - Binfeng Li - conceived the study, and performed the literature search and collected the data.

Email: bflicancer@163.com

Author 2 - Fei Xiong - performed the literature search and collected the data, and assessed the study quality.
Author 3 - Shengzhong Yi - revised the manuscript and language, and assessed the study quality.

Email: 2351627732@qq.com

Author 4 - Sheng Wang - conceived the study, assessed the study quality and revised the manuscript and language.

Email: swangcancer@163.com 